\title{
Neck circumference and future cardiovascular events in a high-risk population-A prospective cohort study
}

\author{
Yingnan Dai, Xiaojing Wan, Xin Li, Enze Jin and Xueqi Li
}

\begin{abstract}
Background: The distribution of adipose tissue has been evaluated in relation to cardiovascular risk factors and biochemical components of the metabolic syndrome. Neck circumference (NC) has been shown to have a strong relationship with cardiovascular disease (CVD) and may be a novel indicator of CVD. The aim of this study was to compare the incidence of CVD events in cohorts with different NC distributions, and to correlate NC with future CVD events and relative mortality.

Methods: A prospective cohort study was performed on 12,151 high-risk cardiology outpatients from 2004 until 2014. Anthropometric parameters like body mass index, NC, waist circumference, and hip circumference were measured at baseline and follow-up and compared in different cohorts with high, medium, and low NC. Fatal and non-fatal CVD events were compared in the follow-up study, and survival analysis was conducted. Independent Chi-square tests were performed to compare the incidence of CVD events and mortality among the cohorts and analyze the interactions.
\end{abstract}

Results: The subjects comprised of 6696 women and 5819 men who completed a mean 8.8-year follow-up. All of the participants had two or more CVD risk factors at baseline. At the end of the study, 4049 CVD events had occurred in 2304 participants. The incidence of non-fatal CVD events was 14.08, 16,65, and $25.21 \%$ in the low-NC, medium-NC, and high-NC cohorts, respectively $(p<0.001)$. The all-cause mortality was $9.77,11.93$, and $19.31 \%$, and CVD mortality, 4.00, 6.29, and $8.01 \%$, respectively $(p<0.001)$. Compared with baseline, the number of CVD risk factors in participants had increased from 2.6, 3.0, and 3.4 to 3.5, 4.1, and 4.7 in the low-, medium-, and high-NC cohorts (34, 36, and $38 \%$ ), respectively. The event-free survival rate was 95.32, 80.15, and $75.47 \%$, respectively.

Conclusions: A higher NC indicated a higher incidence of future fatal and non-fatal CVD events and all-cause mortality in both male and female high-risk participants. CVD risk factors increased more in the higher NC group.

NC as a novel indicator of CVD showed good predictive ability for CVD events and mortality in a high-risk population.

\section{Background}

Body obesity is associated with an increased risk of developing metabolic syndrome, type 2 diabetes (T2DM), and hypertension and is considered a major risk factor for cardiovascular disease (CVD) events and early mortality. Excessive adiposity is involved in the pathogenesis of coronary heart disease (CHD), since it is closely associated with the development of hypertension, dyslipidemia, and T2DM [1].

\footnotetext{
* Correspondence: hmu.xueqili@outlook.com

The Fourth Affiliated Hospital of Harbin Medical University, 37 Yiyuan Street, Habin 150001, China
}

(C) 2016 Dai et al. Open Access This article is distributed under the terms of the Creative Commons Attribution 4.0 International License (http//creativecommons.org/licenses/by/4.0/) which permits unrestricted use, distribution, and reproduction in any medium, provided you give appropriate credit to the original author(s) and the source, provide a link to the Creative Commons license, and indicate if changes were made. The Creative Commons Public Domain Dedication waiver (http://creativecommons.org/publicdomain/zero/1.0/) applies to the data made available in this article, unless otherwise stated.

In addition to obesity, the distribution of body weight is considered to be an important aspect of metabolic syndrome, and is a stronger predictor of cardiovascular risk. The preferential accumulation of body fat in specific regions is related to disease risk [2]. Generally, visceral and subcutaneous fat are associated with insulin resistance [3]. Visceral adipose tissue is a unique pathogenic fatty deposit that is strongly implicated in the pathogenesis of insulin resistance, T2DM, and ischemic heart disease [4], and closely correlated with the risk of CVD. Due to its correspondence with visceral adipose tissue, waist circumference (WC) is widely used to identify central obesity and is 
designated as one of the criteria for metabolic syndrome in several clinical definitions. Previous studies have established the relationship between central obesity and the development of CVD [5, 6].

Recent interest is focused on the possible involvement of adiposity as a linking factor in cardiovascular risk. Through direct paracrine effects, locally acting fat depots may contribute to the complications of obesity, in particular vascular disease [7]. Therefore, cardiovascular risk is conferred by specific fat distribution patterns, particularly upper body adiposity, which is as a strong determinant in the population and more strongly associated with glucose intolerance, hyperinsulinemia, diabetes, hypertriglyceridemia, and gout than is lower-body obesity.

Neck circumference (NC), has been evaluated as an index for the distribution of upper-body subcutaneous adipose tissue in relation to cardiovascular risk factors [8] and insulin resistance, and has also been shown to correlate positively with biochemical components of the metabolic syndrome [9]. NC is a valid marker for identifying obese individuals and correlates well with other anthropometric measurements. NC has been independently correlated with cardio-metabolic risk factors above and beyond their relationships with other adiposity measures $[10,11]$.

However, it is unclear whether NC could predict the incidence of cardiovascular events and mortality. Based on these findings, we hypothesized that NC could predict the incidence of cardiovascular events such as acute coronary syndrome, myocardial infarction, percutaneous coronary intervention, and sudden cardiac death. The aim of this study was to compare the incidence of CVD events in cohorts with different fat distributions and to correlate NC with future CVD events and relative mortality.

\section{Methods}

\section{Participants}

Between February 2004 and December 2006, a total of 18,975 consecutive adults with two or more CVD risk factors who had visited the outpatient clinic of cardiology department in the Fourth Affiliated Hospital of Harbin Medical University were recommended to participate in this study. The definition of CVD risk factors adopted for this study was based on that given by the International Diabetes Federation, which included central obesity based on WC (WC $\geq$ $85 \mathrm{~cm}$ for men; $\mathrm{WC} \geq 80 \mathrm{~cm}$ for women in the Chinese population), with the addition of at least two of the following four factors: increased triglyceride (TG) $(\geq 1.7 \mathrm{mmol} / \mathrm{L})$, decreased high-density lipoprotein cholesterol (HDL-C) ( $\leq 1.03 \mathrm{mmol} / \mathrm{L}$ for men and $\leq 1.29 \mathrm{mmol} / \mathrm{L}$ for women), high blood pressure (BP) ( $\mathrm{SB} \geq 130$ or $\mathrm{DBP} \geq 85 \mathrm{mmHg}$ ), and increased fasting plasma glucose (FPG) $(\geq 5.60 \mathrm{mmol} / \mathrm{L})$ [12]. The Exclusion criteria were as follows: subjects with goiter and other neck masses and deformities; pregnancy; people with severe disabilities or systemic disease, hepatic failure, renal failure; and subjects with diagnosed CVD or T2DM. For all deaths, we sought death certificates, or medical record numbers when appropriate, to understand the cause of death.

After exclusion criteria were applied, 12,151 subjects in total agreed to participate in the longitudinal cohort study, aged 24 to 64 years $(47.9 \pm 17.7$ years, $46.5 \%$ men). The protocol and informed consent documents were approved by the ethics committee of the Fourth Affiliated Hospital of Harbin Medical University. All patients gave written informed consent. Every two years, participants were requested to undergo a clinical examination including blood pressure, blood tests, anthropometric measures, and a self-report medical history review for the mean $8.8 \pm 2.1$ years of follow-up study.

\section{Study design}

Subjects were categorized on the basis of their NC level, according to the Beijing Community Diabetes Study [13]. The $\mathrm{NC}$ categories were as follows: low $\mathrm{NC},<33 \mathrm{~cm}$ for women and $<36 \mathrm{~cm}$ for men; medium $\mathrm{NC}, 33 \leq \mathrm{NC}<37 \mathrm{~cm}$ for women and $36 \leq \mathrm{NC}<40 \mathrm{~cm}$ for men; high $\mathrm{NC}, \geq 37 \mathrm{~cm}$ for women and $\geq 40 \mathrm{~cm}$ for men. BMI was categorized on the basis of the following criteria [14]: normal weight, $\mathrm{BMI}<23.99$; overweight, $24.00 \leq \mathrm{BMI}<28.00 ; \quad$ obese, $\mathrm{BMI} \geq 28.00$. All subjects underwent a complete medical examination, a clinical consultation, and blood laboratory tests. All individuals provided details of their demographic, medical history, and use of medication at baseline. Subsequent visits were conducted within 6 months of the specified time when subjects were having regular outpatient appointments, were hospitalized because of CVD events, or had made an appointment at the clinic. Anthropometric measures and biomarkers were assessed on each visit.

Cardiovascular events such as acute coronary syndrome, myocardial infarction, percutaneous coronary intervention (performed by clinical doctors), and sudden cardiac death, as well as all-cause death (based on clinical documents) were recorded. Incident cardiovascular morbidity and fatal events were validated by reviewing hospital records and the records of attending physicians, and were classified by an external endpoint committee of cardiologists and doctors. Myocardial infarction was defined as either nonfatal acute myocardial infarction or coronary death. Since it is difficult to perform coronary angiograms on every patient, diagnoses were based on hospital records of symptoms, electrocardiographic signs, enzyme levels (creatine kinase, as well as troponin $\mathrm{T}$ or $\mathrm{I}$ ), and necropsy. 


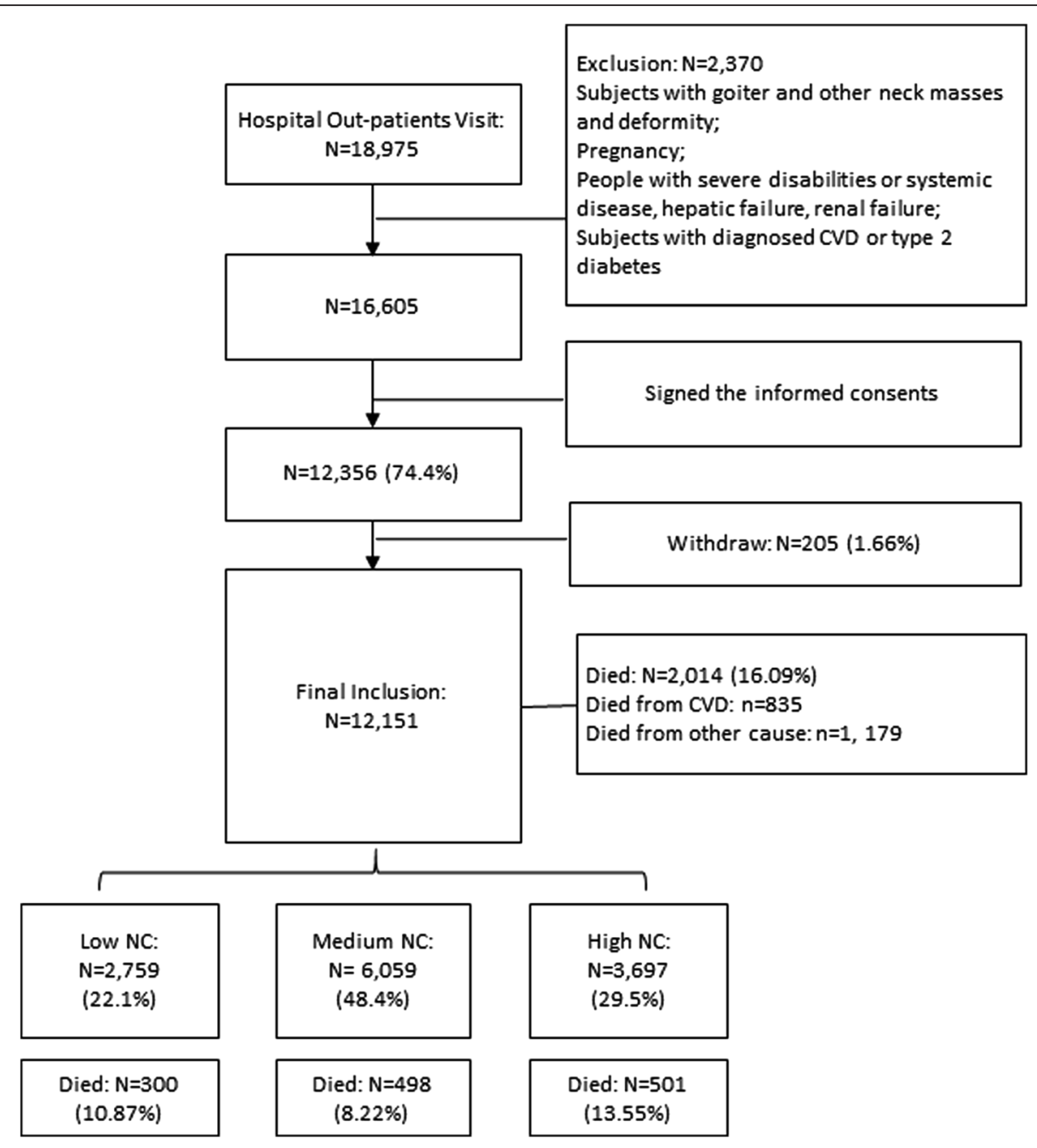

Fig. 1 Flow chart of participants' recruitment

\section{Anthropometric measures}

$\mathrm{NC}(\mathrm{cm})$ was measured with the head erect and eyes facing forward, by using a flexible tape positioned horizontally at the upper margin of the laryngeal prominence. Trained doctors or medical students performed the measurement twice. The average of the two results was adopted. Height and body weight were measured with participants standing without shoes and heavy outer garments. Body weight was measured in light clothing to the nearest $0.1 \mathrm{~kg}$, and height to the nearest $0.5 \mathrm{~cm}$. BMI was calculated as weight (in kilograms) divided by height (in meters) squared. WC was the minimum abdominal girth measured to the nearest $0.1 \mathrm{~cm}$. BP was measured by doctors by using a mercury manometer after the subject had rested for at least $5 \mathrm{~min}$. Three measurements were taken, 5 min apart. The mean of the three measurements was used for the analysis. Hypertension was defined as SBP $\geq 140 \mathrm{mmHg}$ and DBP $\geq 90 \mathrm{mmHg}$.

\section{Biomarkers assessment}

Venous blood samples were drawn from all subjects after they fasted overnight $(10 \mathrm{~h})$. The blood was transferred into glass tubes and allowed to clot at room temperature. Immediately after clotting, the serum was separated by centrifugation at $3000 \mathrm{rpm}$ for $15 \mathrm{~min}$. Plasma glucose was measured by using the hexokinase glucose-6-phosphate dehydrogenase method. The levels of total cholesterol (TC), TG, HDL-C, and low-density lipoprotein cholesterol (LDL-C) were determined enzymatically by using an auto-analyzer (Type C8000; Roche Ltd, Germany). Hemoglobin A1c (HbA1c) was measured by using high performance liquid chromatography (HPLC; HLC-723G7 hemoglobin HPLC analyzer, Tosoh Corp.) according to the standardized method.

The 1999 World Health Organization diagnostic criteria were used to diagnose diabetes. National Cholesterol Education Programme guidelines were used to define dyslipidemia as follows [15]: hypercholesterolemia, serum cholesterol 
Table 1 Characteristics of participants at baseline

\begin{tabular}{|c|c|c|c|}
\hline & Women & Men & $p$ value \\
\hline & $n=6696$ & $n=5819$ & \\
\hline Age (years) & $51.05 \pm 15.67$ & $44.29 \pm 18.1$ & 0.001 \\
\hline BMI $\left(\mathrm{kg} / \mathrm{m}^{2}\right)$ & $26.85 \pm 5.25$ & $26.09 \pm 3.75$ & NS \\
\hline $\mathrm{NC}(\mathrm{cm})$ & $36.31 \pm 7.84$ & $38.19 \pm 5.94$ & NS \\
\hline WC (cm) & $84.51 \pm 8.56$ & $87.96 \pm 12.56$ & NS \\
\hline $\mathrm{HC}(\mathrm{cm})$ & $99.1 \pm 8.66$ & $94.66 \pm 12.68$ & $<0.001$ \\
\hline WHR & $0.89 \pm 0.08$ & $0.97 \pm 0.06$ & $<0.001$ \\
\hline FPG (mmol/L) & $6.16 \pm 1.39$ & $6.86 \pm 1.31$ & NS \\
\hline HbA1c (\%) & $5.92 \pm 0.67$ & $6.13 \pm 0.98$ & NS \\
\hline TC (mmol/L) & $5.51 \pm 1.19$ & $5.29 \pm 1.09$ & NS \\
\hline TG (mmol/L) & $1.79 \pm 0.98$ & $2.01 \pm 1.19$ & $<0.001$ \\
\hline HDL-C (mmol/L) & $1.53 \pm 0.35$ & $1.33 \pm 0.26$ & NS \\
\hline LDL-C (mmol/L) & $3.51 \pm 1.08$ & $3.48 \pm 0.88$ & NS \\
\hline $\mathrm{SBP}(\mathrm{mm} \mathrm{Hg})$ & $135.71 \pm 15.14$ & $133.41 \pm 16.84$ & NS \\
\hline $\mathrm{DBP}(\mathrm{mm} \mathrm{Hg})$ & $77.66 \pm 8.47$ & $78.96 \pm 8.65$ & NS \\
\hline Smoking (n, \%) & 2690 (40.17\%) & 3547 (60.96 \%) & NS \\
\hline Alcohol drinking (n, \%) & 893 (13.34 \%) & 985 (16.93 \%) & NS \\
\hline
\end{tabular}

Values represent mean $\pm \mathrm{SD}, \mathrm{n}(\%)$. A $p$ value $\leq 0.05$ was considered statistically significant. NS not significant $(p>0.05)$; BMI body mass index; NC neck circumference; WC waist circumference; $H C$ hip circumference; WHR waist-hip ratio; FPG fasting plasma glucose; HbA1C hemoglobin A1c; TC total cholesterol; $T G$ triglyceride; $H D L-C$ high-density lipoprotein cholesterol; $L D L-C$ low-density lipoprotein cholesterol; SBP systolic blood pressure; DBP diastolic blood pressure

levels $\geq 200 \mathrm{mg} / \mathrm{dL}(\geq 5.2 \mathrm{mmol} / \mathrm{L})$; hypertriglyceridemia, serum TG levels $\geq 150 \mathrm{mg} / \mathrm{dL}(\geq 1.7 \mathrm{mmol} / \mathrm{L})$; low HDL cholesterol, HDL cholesterol levels $<40 \mathrm{mg} / \mathrm{dL}$ $(<1.04 \mathrm{mmol} / \mathrm{L})$ for men and $<50 \mathrm{mg} / \mathrm{dL}(<1.3 \mathrm{mmol} / \mathrm{L})$ for women; high LDL cholesterol, and LDL cholesterol levels $\geq 130 \mathrm{mg} / \mathrm{dL}(\geq 3.4 \mathrm{mmol} / \mathrm{L})$.

\section{Statistical analysis}

All data were analyzed by using SPSS 17.0 software. All continuous data are presented as mean \pm standard deviation (SD) or percentages. Chi-square tests were used to examine differences between categorical data at baseline and follow-up. Changes over the 8.8-year follow-up period were assessed by using repeated measures ANOVA. Data were tested for equality of variance, and a correction for unequal variances was performed when appropriate. All tests of significance were two tailed. Statistical significance was based on a $p$ value of $<0.05$.

\section{Results}

There were 18, 975 subjects who visited the outpatient clinic of the cardiology department of the Fourth Affiliated Hospital of Harbin Medical University in search of help for a cardiovascular problem. After an initial screening, we excluded those whose results did not meet the inclusion criteria $(n=2370)$. Of the patients who were invited to participate in this study, $74.4 \%$ agreed and provided informed consent. During the average 8.8 years of follow-up, 205 subjects withdrew because of migration or loss of contact. Subjects in our study were assigned to one of three cohorts according to their $\mathrm{NC}$ levels. There were 2757, 6059, and 3697 participants included in the low, medium, and high NC groups, respectively. At the end of the follow-up study, 10.87, 8.22, and $13.55 \%$ had died from all causes in these three cohorts, respectively (Fig. 1).

Demographic characteristics and CVD risk factors are displayed in Table 1 . The study sample consisted of 12,515 individuals, with 6696 (53.50\%) women and 5819 men, a mean age of $47.9 \pm 17.7$ years, and BMI of $26.31 \pm 4.48$. All the participants had two or more CVD risk factors. Except for age, $\mathrm{HC}$, WHR, and TG, the other variables were not significantly different between the sexes. Both the sex groups had high levels of FGP, TC, TG, LDL, and a high percentage of smoking and alcohol consumption at baseline. Of the 12,515 subjects, the metabolic syndrome was diagnosed in 2113 (18.66 \%), high BP in 4928 (39.38\%), increased FPG in 1872 (14.96\%), increased TG in 4975 (39.75\%), decreased HDL in 3487 (27.86 \%), increased LDL in 6711 (53.62\%), and increased TC in 8361 (66.81\%) participants.

At the end of the follow-up study, 4049 CVD events had occurred in 2304 participants. The incidence of non-fatal CVD events was $14.08 \%$ in the low-NC cohort, $16.65 \%$ in the medium-NC cohort, and $25.21 \%$ in the high-NC cohort $(p<0.001)$. The all-cause mortality was 9.77, 11.93, and $19.31 \%$, and CVD mortality was $4.00,6.29$ and $8.01 \%$, respectively $(p<0.001)$. After a mean follow-up of 8.8 years (90,605 person-years), we observed 1404 nonfatal CVD events in 859 men and 645 women, and 2014 deaths (835 CVD deaths, $41.5 \%$ ) in 1175 men (505 CVD deaths, $43.0 \%$ ) and 839 women (430 CVD deaths, $51.0 \%$ ).

When categorized by $\mathrm{NC}$ into three groups, an increased risk level for CVD with increased NC was observed (Fig. 2). The interaction between NC and BMI on the incidence of CVD events, all-cause mortality, and CVD mortality were also analyzed. Within each NC group, there was a stepwise increase in the incidence of CVD events, all-cause mortality, and CVD mortality in men and women $(p<0.001)$. Among the men, there was a significant interaction between $\mathrm{NC}$ and the incidence of CVD events $(p<0.005)$, all-cause mortality $(p<0.001)$, and CVD mortality $(p<0.05)$. Among the women, a significant interaction was also seen between $\mathrm{NC}$ and the incidence of CVD events $(p<0.05)$, all-cause mortality $(p<0.001)$, and CVD mortality $(p<0.001)$. After a mean 8.8-year followup, 4049 CVD events had occurred among 2304 participants at baseline, including 1259 women (incidence $18.8 \%$ ), and 1045 men (incidence $17.96 \%$ ). Despite the presence of CVD events, in all the 12,515 participants at 


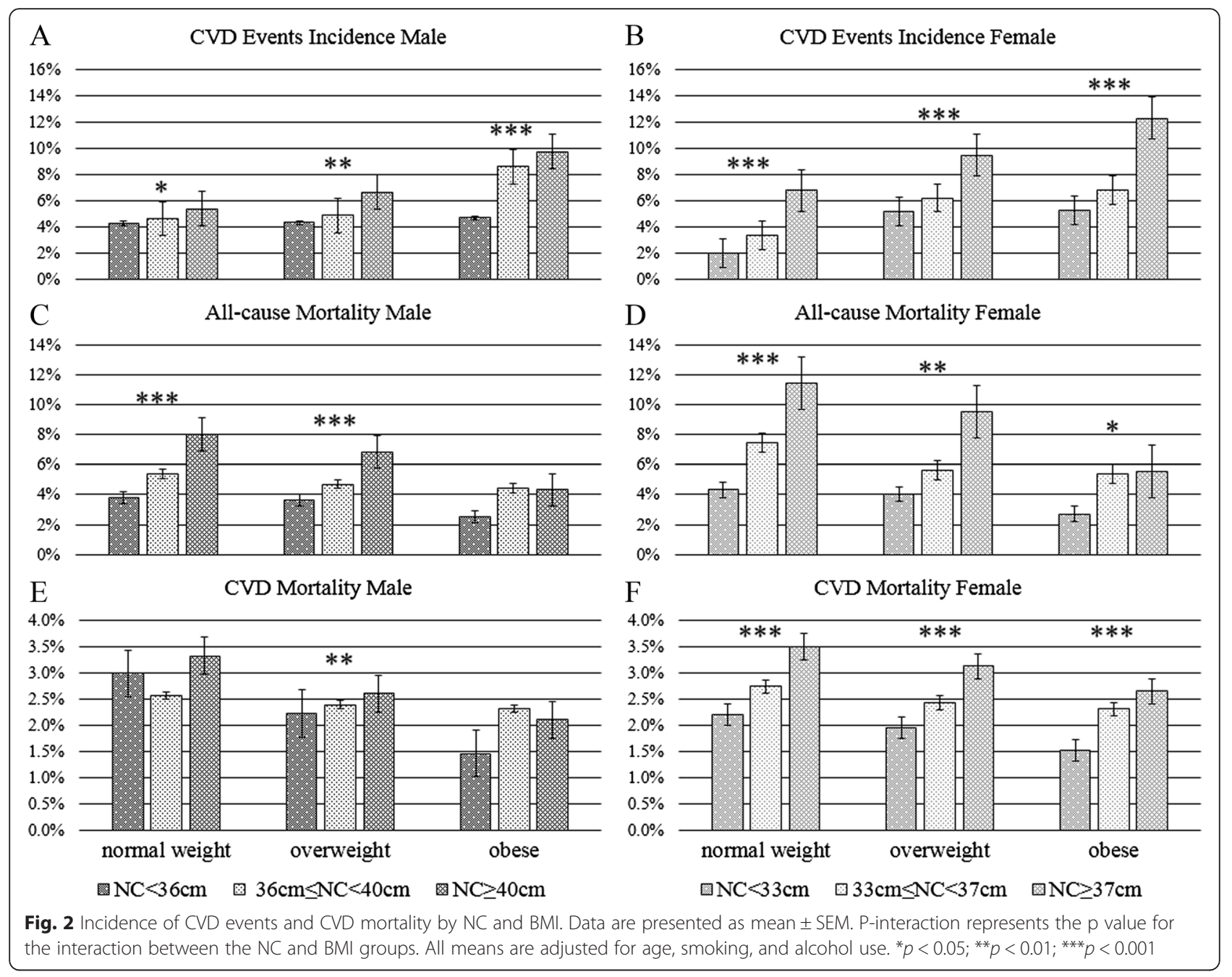

baseline, all-cause mortality at 8.8-year follow-up was $16.09 \%(n=2014)$, including $17.55 \%(n=1175)$ in women and $14.42 \%(n=839)$ in men. There were 935 deaths caused by CVD (mortality $7.47 \%$ ), in 505 women (mortality $7.54 \%)$ and 430 men (7.39\%). In the high-NC group, BMI showed a more significant influence on CVD events and mortality. Likewise, in the high BMI group, NC also significantly influenced the CVD outcomes.

Differences in CVD risk levels among NC groups between baseline and follow-up are listed in Table 2. At baseline, significant differences among the $\mathrm{NC}$ groups were found for BMI $(p=0.05)$, NC $(p<0.001)$, WC $(p=0.001)$, HC $(p<0.001)$, WHR $(p<0.001)$, FPG $(p=0.004)$, TG $(p<0.001)$, HDL-C $(p<0.001)$, and DBP $(p=0.039)$. In all groups, $\mathrm{BMI}$ increased significantly between baseline and follow-up $(p<0.001)$, as well as WC $(p<0.001)$, WHR $(p<0.001)$, FPG $(p=0.032)$, TG $(p=0.027)$, TC $(p=0.019)$, HDL-C ( $p=0.019)$, LDL-C ( $p=0.034)$, and SBP $(p=0.048)$. However, age, TC, LDL-C, and SBP did not show significant differences among NC groups at baseline $(p=0.632$,
0.082, 0.116, and 0.137, respectively). NC, HC, and DBP did not change significantly between baseline and followup ( $p=0.051,0.127$, and 0.166, respectively).

Compared with baseline, the number of CVD risk factors in participants had increased from 2.6, 3.0, and 3.4 to $3.5,4.1$, and 4.7 in the low-, medium-, and high-NC cohorts (34, 36 and $38 \%$ ). The event-free survival rate was 95.32, 80.15, and $75.47 \%$. CVD risk factors and event free survival by $\mathrm{NC}$ group in years are shown in Fig. 3. Every 2 years, we calculated the number of CVD risk factors. The CVD risk factors increased in number with $\mathrm{NC}$ in the mean 8.8 years of follow-up, with subjects in the high-NC group having 1.2 times as many risk factors as those of participants the low-NC group at the end of follow-up (at baseline, 1.4 times). CVD-event-free survival also decreased in the 8.8 years of follow-up, with subjects in the low-risk group having a $20 \%$ higher CVD-eventfree survival rate than that of those in the high $\mathrm{NC}$ group $(p<0.01)$. 
Table 2 Differences in CVD risk levels among NC groups between baseline and follow-up

\begin{tabular}{|c|c|c|c|c|c|c|c|c|}
\hline & \multicolumn{2}{|l|}{$\mathrm{NC}<36 \mathrm{~cm}$} & \multicolumn{2}{|c|}{$36 \mathrm{~cm} \leq \mathrm{NC}<40 \mathrm{~cm}$} & \multicolumn{2}{|l|}{$\mathrm{NC} \geq 40 \mathrm{~cm}$} & \multicolumn{2}{|c|}{$p$ values } \\
\hline & Baseline & Follow-up & Baseline & Follow-up & Baseline & Follow-up & Time & Group \\
\hline $\bar{n}$ & $n=2759$ & $n=2282$ & $n=6059$ & $n=5254$ & $n=3697$ & $n=2760$ & - & - \\
\hline Age (year) & $46.33 \pm 18.6$ & $56.65 \pm 19.12$ & $48.64 \pm 17.84$ & $57.42 \pm 18.81$ & $47.96 \pm 16.58$ & $54.56 \pm 16.28$ & - & 0.632 \\
\hline BMI $\left(\mathrm{kg} / \mathrm{m}^{2}\right)$ & $24.53 \pm 5.47$ & $28.4 \pm 5.91$ & $26.48 \pm 4.75$ & $30.4 \pm 3.86$ & $28.18 \pm 6.92$ & $30.34 \pm 6.12$ & $<0.001$ & 0.05 \\
\hline $\mathrm{NC}(\mathrm{cm})$ & $31.91 \pm 4.2$ & $32.5 \pm 4.45$ & $37.67 \pm 1.58$ & $37.23 \pm 1.73$ & $41.23 \pm 1.21$ & $41.55 \pm 1.45$ & 0.051 & $<0.001$ \\
\hline WC $(\mathrm{cm})$ & $83.26 \pm 10.33$ & $84.34 \pm 11.64$ & $86.8 \pm 9.72$ & $87.54 \pm 10.73$ & $87.21 \pm 10.96$ & $89.54 \pm 12.75$ & $<0.001$ & 0.001 \\
\hline $\mathrm{HC}(\mathrm{cm})$ & $92.71 \pm 10.43$ & $92.53 \pm 12.85$ & $96.94 \pm 9.22$ & $97.22 \pm 9.6$ & $100.55 \pm 10.53$ & $103.65 \pm 10.35$ & 0.127 & $<0.001$ \\
\hline WHR & $0.88 \pm 0.14$ & $0.89 \pm 0.16$ & $0.92 \pm 0.13$ & $0.93 \pm 0.15$ & $0.97 \pm 0.13$ & $0.99 \pm 0.16$ & $<0.001$ & $<0.001$ \\
\hline $\mathrm{FPG}(\mathrm{mmol} / \mathrm{L})$ & $6.28 \pm 1.33$ & $6.25 \pm 1.34$ & $6.46 \pm 1.26$ & $6.59 \pm 1.22$ & $6.68 \pm 1.29$ & $6.77 \pm 1.28$ & 0.032 & 0.004 \\
\hline HbA1c (\%) & $5.78 \pm 0.84$ & $5.98 \pm 1.05$ & $5.98 \pm 0.67$ & $6.12 \pm 0.73$ & $6.25 \pm 1.02$ & $6.42 \pm 1.35$ & 0.048 & 0.137 \\
\hline TG (mmol/L) & $1.72 \pm 0.94$ & $1.78 \pm 1.08$ & $1.9 \pm 0.91$ & $1.95 \pm 0.96$ & $2 \pm 0.94$ & $2.15 \pm 1.11$ & 0.027 & $<0.001$ \\
\hline TC (mg/dL) & $5.17 \pm 1.17$ & $5.32 \pm 1.01$ & $5.43 \pm 1.15$ & $5.67 \pm 1.74$ & $5.54 \pm 1.67$ & $5.73 \pm 1.83$ & 0.019 & 0.082 \\
\hline $\mathrm{HDL}-\mathrm{C}(\mathrm{mg} / \mathrm{dL})$ & $1.67 \pm 0.37$ & $1.65 \pm 0.27$ & $1.43 \pm 0.34$ & $1.25 \pm 0.36$ & $1.27 \pm 0.29$ & $1.13 \pm 0.36$ & 0.006 & $<0.001$ \\
\hline LDL-C (mg/dL) & $3.17 \pm 0.88$ & $3.26 \pm 0.93$ & $3.57 \pm 0.95$ & $3.62 \pm 1.02$ & $3.61 \pm 0.86$ & $3.96 \pm 1.01$ & 0.034 & 0.116 \\
\hline SBP (mm Hg) & $133.62 \pm 16.78$ & $136.9 \pm 15.48$ & $134.63 \pm 14.95$ & $137.21 \pm 15.17$ & $135.42 \pm 14.96$ & $140.07 \pm 16.39$ & 0.048 & 0.137 \\
\hline $\mathrm{DBP}(\mathrm{mm} \mathrm{Hg})$ & $76.27 \pm 8.46$ & $76.2 \pm 7.7$ & $77.55 \pm 8.15$ & $77.34 \pm 7.98$ & $80.92 \pm 8.95$ & $81.11 \pm 9.35$ & 0.166 & 0.039 \\
\hline
\end{tabular}

\section{Discussion}

To the best of our knowledge, this is the first prospective cohort study to evaluate the association between $\mathrm{NC}$ and future CVD events and mortality and the first Chinese population cohort to be assessed for an association between fat distribution and CVD mortality. In the present prospective study of a large cohort of Chinese people at high risk of CVD, we examined the incidence of cardiovascular events and mortality in people with different fat distributions (measured by $\mathrm{NC}$ and $\mathrm{BMI}$ ). First, we observed that $\mathrm{NC}$ is associated with cardiometabolic risk factors. Second, a higher NC indicated a higher incidence of CVD events in the future. Third, a higher NC was associated with higher all-cause and CVD mortality.

Regional variations in adipocyte secretion have been observed to make differential contributions to disease risk. Subcutaneous fat and peripheral body fat stored predominantly in the femoral, gluteal, and thigh regions help protect against ectopic fat accumulation. Consequently, different accumulations of fat play different roles in obesity-related diseases like insulin resistance, dyslipidemia, and fatty liver. Britton et al. found that visceral adiposity was associated with incident CVD and even cancer [16]. In the Heinz Nixdorf Recall Study, epicardial fat was proved be associated with fatal and nonfatal coronary events, including CVD risk factors and incident myocardial infarction in the general population [17]. More recently, fat accumulation in not only the visceral region but also other regions such as the liver, muscle, pericardial, perivascular, and even perirenal areas was found to be associated with CVD and heart function [18-22].
Free fatty acid release from upper-body subcutaneous fat was reported to be higher than that from lower-body subcutaneous fat [23]. In recent years, several studies have examined the association between $\mathrm{NC}$ and cardiometabolic risk factors. One of the most famous among these studies was the Framingham Heart Study, which demonstrated that $\mathrm{NC}$ is associated with CVD risk factors even after adjustment for visceral adipose tissue and BMI, and considered NC to be a novel measure of cardiometabolic risk [11]. The effectiveness of $\mathrm{NC}$ has been demonstrated several times in the Chinese population. In the Beijing Community Diabetes Study 4, NC was positively associated with the metabolic syndrome in Chinese people with T2DM [13]. In a study by Zhou, $\mathrm{NC}$ was significantly correlated with all outcomes of cardio-metabolic risk in both sexes [24]. In the Cardiometabolic Risk in Chinese study of a population of apparently healthy Chinese adults, significant associations were found between high NC and increased risk of insulin resistance and various CVD risk factors. These researchers hold the viewpoint that $\mathrm{NC}$ could identify those at high risk of CVD and T2DM [25]. Our results were in line with these previous studies and other studies $[9,26]$. In other words, the higher the $\mathrm{NC}$, the greater the risk that cardio-metabolic disturbances will develop in the future. Our findings indicate that NC may be a valid and powerful indicator of CVD.

Although a number of epidemiological studies have demonstrated that diversity in body fat distribution significantly predicts premature death, $\mathrm{NC}$ had been used as a novel anthropometric measure of upper-trunk 


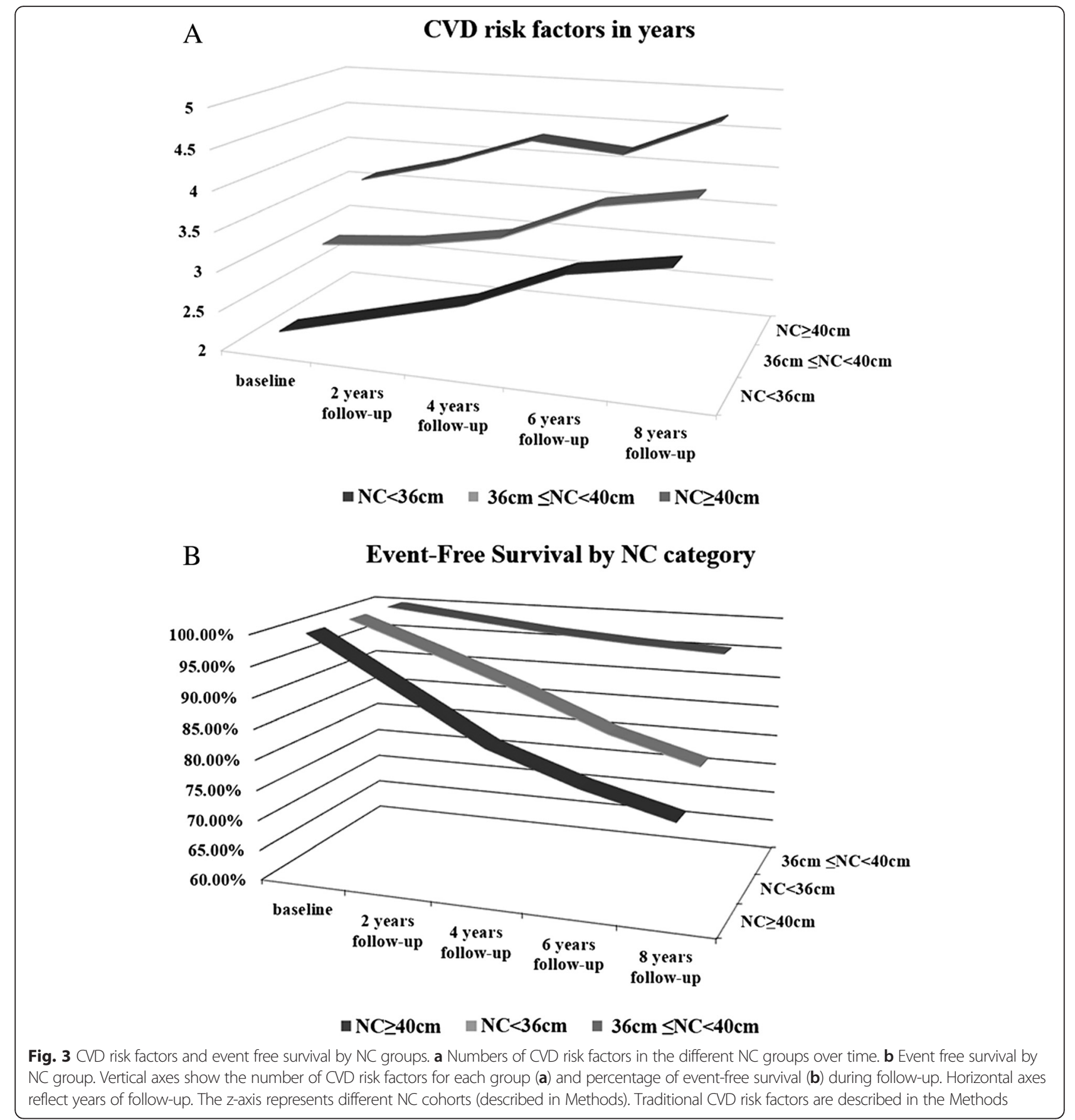

body fat, but had not been assessed for its ability to predict mortality, especially surprising considering its strong associations with CVD risk. The use of BMI combined with measures of central obesity was analyzed recently to assess mortality in subjects with coronary disease [27]. The researchers focused on the role of central obesity in people with normal weight and found that normal weight with central obesity was associated with the highest risk of mortality. However, although NC measurements reduced the shortcomings of WC measurements, which are easily affected by meals, breathing, and clothing, $\mathrm{NC}$ was not considered in that study.

This current study is the first prospective study of $\mathrm{NC}$ to evaluate its predictive ability for CVD events and mortality. Recent studies have proven the predictive ability of other indicators like $\mathrm{WC}, \mathrm{HC}$, and WHR [28-32]; however, the results seemed inconsistent. In the Norfolk cohort, Canoy et al. conducted a population-based prospective study to 
investigate body fat distribution and the risk of CHD. They compared WC, WHR, and BMI and found that abdominal obesity was more consistently and strongly predictive of CHD than BMI, especially with respect to the risk of obesity-related CHD [33]. WHR, WC, and even BMI were influenced by possible biological factors and confounders. Although directly related to the development of CVD, these indices still confuse clinicians when deciding how they can best predict future events. In our study, NC strongly affected CVD events and mortality, even in the normal BMI group. Because it is not affected by BMI, NC showed a more stable association with CVD.

When the associations with BMI and $\mathrm{NC}$ were assessed separately, a higher NC and BMI was associated with a higher incidence of CVD events, but for any given $\mathrm{NC}$, those with a larger BMI had a lower mortality than those with a smaller BMI, regardless of CVD mortality. In the general population, BMI in the overweight and obese ranges has been associated with higher overall mortality [34]. In our findings, BMI remained predictive of CVD and CVD mortality. By contrast, a high BMI was also found to contribute to lower mortality in those with CVD and even in other populations, as observed in many studies [35-37]. Furthermore, BMI has been demonstrated to have a suboptimal correlation with body fat, especially in patients with CVD, because slightly overweight or obese individuals can vary in body fat distribution and associated metabolic risk factors $[18,38]$. It suggested to us that some individuals with a high BMI but a low NC may be a typo among our participants, and even those with a low BMI but a high NC. Our results provided more evidence to support the obesity paradox and were consistent with previous conclusions. $\mathrm{NC}$ could be a superior predictor of mortality in the population at high-risk of CVD. Compared with BMI, $\mathrm{NC}$ is not influenced by these paradoxes and showed a stable ability to predict mortality.

To see the influence of sex on the results, we analyzed the data for both sexes when comparing the interactions of BMI and NC. Both BMI and NC had different impacts on the incidence of CVD events, and also CVD or all-cause mortality in men and women, but to a different degree (Fig. 2). We found increased risks associated with a higher NC value, but with different trends in men and women. A possible explanation for this phenomenon is that there is a greater delivery of free fatty acid from visceral adipose tissue to the liver in women than men [39] and free fatty acid is considered to be one of the most important risk factors for CVD. However, all the interactions were significant for both sexes $(p<0.05)$.

\section{Strengths and limitations}

The main strength of the present study is that we firstly evaluated $\mathrm{NC}$ and its association with future CVD outcomes in a high-risk population, with the cohort of individuals manifesting both $\mathrm{NC}$ and $\mathrm{BMI}$ variability. Furthermore, as this was designed as a prospective study, we succeeded in attaining a high rate of follow-up and minimized the possibility of bias resulting from loss of follow-up. In addition, comparisons between men and women within the same cohort have been scarce or not reported adequately. When comparing the interactions of BMI and NC, we calculated the data for both sexes. Therefore, we could see the influence of sex on the results. The large sample size of the study increased the precision and provided us with extensive information that allowed us to control for potential confounders.

Potential limitations of this study were the lack of comparisons for all anthropometric measures such as WC, $\mathrm{HC}$, and WHR. To consider all these variables together, large-scale population-based studies are needed. A second limitation is the absence of consideration of all the interactions between risk factors like blood pressure or lipids. Although they form part of the causal pathway, taking these factors into account could help toward understanding their role as mediating factors. Further studies are needed to identify the relationship between $\mathrm{NC}$ and fatal and nonfatal CVD events in the general population.

\section{Conclusions}

$\mathrm{NC}$ is associated with the future incidence of CVD events, mortality, and all-cause mortality, particular among men and women who have risk factors. A graded relationship between NC and CVD events persisted in a population at high risk of CVD. A higher NC indicated a higher incidence of CVD events, mortality, and all-cause mortality in the future. NC could be a superior, simpler, and more consistent indicator of CVD and related mortality in the highCVD-risk population.

\section{Abbreviations}

BMI: body mass index; BP: blood pressure; CHD: coronary heart disease; CVD: cardiovascular disease; DBP: diastolic blood pressure; FPG: fasting plasma glucose; HbA1c: hemoglobin A1c; HC: hip circumference; HDLC: high-density lipoprotein cholesterol; HPLC: high performance liquid chromatography; LDL-C: low-density lipoprotein cholesterol; NC: neck circumference; SBP: systolic blood pressure; TC: total cholesterol;

TG: triglyceride; T2DM: type 2 diabetes mellitus; WC: waist circumference; WHR: waist-hip ratio.

\section{Competing interests}

The authors declare that they have no competing interests.

\section{Authors' contributions}

YD carried out the study design, participated in the data analysis, and drafted the manuscript. XW and Xin L carried out the data collection and helped to draft the manuscript. EJ performed the statistical analysis, and participated in the design of the study. Xueqi $L$ conceived the study, participated in its design and coordination, and helped to draft the manuscript. All authors read and approved the final manuscript. 
Received: 21 September 2015 Accepted: 2 March 2016

Published online: 05 March 2016

\section{References}

1. Logue J, et al. Obesity is associated with fatal coronary heart disease independently of traditional risk factors and deprivation. Heart (British Cardiac Society). 2011;97:564-8. doi:10.1136/hrt.2010.211201.

2. Santosa S, Jensen MD. Why are we shaped differently, and why does it matter? American journal of physiology Endocrinology and metabolism. 2008;295:E531-535. doi:10.1152/ajpendo.90357.2008.

3. Ramachandran A, Wan Ma RC, Snehalatha C. Diabetes in Asia. The Lancet. 2010;375:408-18. http://dx.doi.org/10.1016/S0140-6736(09)60937-5.

4. Kuk J , et al. Visceral fat is an independent predictor of all-cause mortality in men. Obesity (Silver Spring, Md). 2006;14:336-41. doi:10.1038/oby.2006.43.

5. Pemberton $V L$, et al. Report of the National Heart, Lung, and Blood Institute's Working Group on obesity and other cardiovascular risk factors in congenital heart disease. Circulation. 2010;121:1153-9. doi:10.1161/ circulationaha.109.921544.

6. Ginsberg HN, MacCallum PR. The obesity, metabolic syndrome, and type 2 diabetes mellitus pandemic: Part I. Increased cardiovascular disease risk and the importance of atherogenic dyslipidemia in persons with the metabolic syndrome and type 2 diabetes mellitus. Journal of the cardiometabolic syndrome. 2009;4:113-9. doi:10.1111/j.1559-4572.2008.00044.x

7. Eringa EC, Bakker W, van Hinsbergh WW. Paracrine regulation of vascular tone, inflammation and insulin sensitivity by perivascular adipose tissue. Vascular pharmacology. 2012;56:204-9. doi:10.1016/j.vph.2012.02.003.

8. Ben-Noun LL, Laor A. Relationship between changes in neck circumference and cardiovascular risk factors. Experimental and clinical cardiology. 2006;11:14-20.

9. Onat $\mathrm{A}$ et al. Neck circumference as a measure of central obesity: associations with metabolic syndrome and obstructive sleep apnea syndrome beyond waist circumference. Clinical nutrition. 2009:28:46-51.

10. Fitch KV, Stanley TL, Looby SE, Rope AM, Grinspoon SK. Relationship between neck circumference and cardiometabolic parameters in HIVinfected and non-HIV-infected adults. Diabetes care. 2011;34:1026-31.

11. Preis SR, et al. Neck circumference as a novel measure of cardiometabolic risk: the Framingham Heart study. The Journal of Clinical Endocrinology \& Metabolism. 2010:95:3701-10.

12. Alberti K, Zimmet P, Shaw J. Metabolic syndrome-a new world-wide definition. A consensus statement from the international diabetes federation Diabetic Medicine. 2006;23:469-80.

13. Yang G-R, et al. Neck circumference positively related with central obesity, overweight, and metabolic syndrome in Chinese subjects with type 2 diabetes: Beijing Community Diabetes Study 4. Diabetes care. 2010;33:2465-7

14. Chen C, Lu F, Chen C, Lu FC. Obesity: The criteria in the Guidelines for Prevention and Control of Overweight and Obesity in Chinese Adult (Excerpt). Biomedical and environmental sciences: BES. 2004:17:1.

15. Detection EPO. EVALUATION, AND TREATMENT OF HIGH BLOOD CHOLESTEROL IN ADULTS. Executive summary of the third report of The National Cholesterol Education Program (NCEP) Expert panel on detection, evaluation, and treatment of high blood cholesterol in adults (Adult Treatment Panel III). Jama. 2001;285:2486-97.

16. Britton $\mathrm{KA}$, et al. Body fat distribution, incident cardiovascular disease, cancer, and all-cause mortality. Journal of the American College of Cardiology. 2013;62:921-5.

17. Mahabadi AA et al. Association of epicardial fat with cardiovascular risk factors and incident myocardial infarction in the general population: the Heinz Nixdorf Recall Study. Journal of the American College of Cardiology. 2013;61:1388-95.

18. Lim S. Ectopic Fat Assessment Focusing on Cardiometabolic and Renal Risk. Endocrinology and Metabolism. 2014;29:1-4.

19. Wang CY, Ou HY, Chen MF, Chang TC, Chang CJ. Enigmatic Ectopic Fat: Prevalence of Nonalcoholic Fatty Pancreas Disease and Its Associated Factors in a Chinese Population. Journal of the American Heart Association. 2014:3:e000297.

20. Okauchi $Y$, et al. Reduction of visceral fat is associated with decrease in the number of metabolic risk factors in Japanese men. Diabetes Care. 2007;30:2392-4

21. Hiuge-Shimizu A, et al. Reduction of visceral fat correlates with the decrease in the number of obesity-related cardiovascular risk factors in Japanese with Abdominal Obesity (VACATION-J Study). Journal of atherosclerosis and thrombosis. 2011;19:1006-18.
22. Neeland IJ, et al. Relation of regional fat distribution to left ventricular structure and function. Circulation: Cardiovascular Imaging. 2013;6:800-7.

23. Guo Z, Hensrud DD, Johnson CM, Jensen MD. Regional postprandial fatty acid metabolism in different obesity phenotypes. Diabetes. 1999;48:1586-92.

24. Zhou J, et al. Neck circumference as an independent predictive contributor to cardio-metabolic syndrome. Cardiovasc Diabetol. 2013;12:76.

25. Liang J, et al. Neck Circumference and Insulin Resistance in Chinese Adults: The Cardiometabolic Risk in Chinese (CRC) Study. Diabetes Care. 2013:36:e145-6.

26. Stabe $C$, et al. Neck circumference as a simple tool for identifying the metabolic syndrome and insulin resistance: results from the Brazilian Metabolic Syndrome Study. Clinical endocrinology. 2012;78:874-81.

27. Coutinho T, et al. Combining Body Mass Index With Measures of Central Obesity in the Assessment of Mortality in Subjects With Coronary DiseaseRole of "Normal Weight Central Obesity". Journal of the American College of Cardiology. 2013;61:553-60.

28. Heitmann BL, Frederiksen P, Lissner L. Hip circumference and cardiovascular morbidity and mortality in men and women. Obesity research. 2004;12:482-7.

29. Welborn TA, Dhaliwal SS, Bennett SA. Waist-hip ratio is the dominant risk factor predicting cardiovascular death in Australia. Medical Journal of Australia. 2003;179:580-5.

30. Li TY, et al. Obesity as compared with physical activity in predicting risk of coronary heart disease in women. Circulation. 2006:113:499-506.

31. Lakka H-M, Lakka TA, Tuomilehto J, Salonen JT. Abdominal obesity is associated with increased risk of acute coronary events in men. European heart journal. 2002;23:706-13.

32. Yusuf $\mathrm{S}$, et al. Obesity and the risk of myocardial infarction in 27000 participants from 52 countries: a case-control study. The Lancet. 2005; 366:1640-9.

33. Canoy D, et al. Body Fat Distribution and Risk of Coronary Heart Disease in Men and Women in the European Prospective Investigation Into Cancer and Nutrition in Norfolk Cohort A Population-Based Prospective Study. Circulation. 2007;116:2933-43.

34. Collaboration PS. Body-mass index and cause-specific mortality in 900000 adults: collaborative analyses of 57 prospective studies. The Lancet. 2009; 373:1083-96.

35. Romero-Corral A, et al. Association of bodyweight with total mortality and with cardiovascular events in coronary artery disease: a systematic review of cohort studies. The Lancet. 2006;368:666-78.

36. Lavie CJ, et al. Body composition and survival in stable coronary heart disease: impact of lean mass index and body fat in the "obesity paradox". Journal of the American College of Cardiology. 2012;60:1374-80.

37. Lavie CJ, Milani RV, Ventura HO. Impact of Obesity on Outcomes in Myocardial InfarctionCombating the "Obesity Paradox". Journal of the American College of Cardiology. 2011;58:2651-3.

38. Romero-Corral A, et al. Diagnostic performance of body mass index to detect obesity in patients with coronary artery disease. European heart journal. 2007;28(17):2087-93.

39. Nielsen S, Guo Z, Johnson CM, Hensrud DD, Jensen MD. Splanchnic lipolysis in human obesity. The Journal of clinical investigation. 2004;113:1582-8.

\section{Submit your next manuscript to BioMed Central and we will help you at every step:}

- We accept pre-submission inquiries

- Our selector tool helps you to find the most relevant journal

- We provide round the clock customer support

- Convenient online submission

- Thorough peer review

- Inclusion in PubMed and all major indexing services

- Maximum visibility for your research

Submit your manuscript at www.biomedcentral.com/submit 